an abundance of good hardwood already in the country, and the four pitch-pines, rivalling hardwoods in strength and durability. Eucalypt culture in America is still in its infancy; they have not yet discriminated the valuable from the many worthless species, nor fitted, as far as may be, the species to its climate.

D. E. Hutchins.

Cape Town, June 23.

E. Hurchins.

\section{A Simple Form of Tide Predictor.}

FOR the past four years a very simple form of tide-predicting machine, the invention of Captain A. Inglis, the harbour-master, has been in use at Port Adelaide for the construction of the yearly published tide tables. The tides at Port Adelaide are rather peculiar in their behaviour, this being due principally to the fact that the solar and lunar semi-diurnal components are almost exactly equal. At and near the neaps these neutralise one another, and the diurnal components, which are relatively large, are then the main sources of the tidal movement. Before these tides were harmonically analysed, their prediction by ordinary methods was quite impossible, except near the springs. By means of this machine, however, they are now predicted yearly with considerable accuracy. The essential principles of the machine are as follows:-A number of thin wooden templets are cut, each in the form of a sine curve, representing the various tidal components (Fig. I). These waves are of different lengths, the length of each component wave bearing

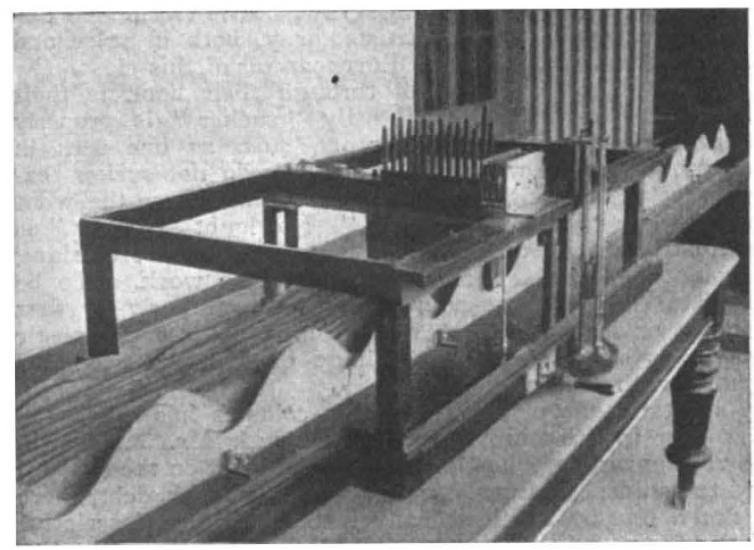

FiG. I.

the same ratio to the solar semi-diurnal as its angular speed does to $15^{\circ}$. The templets are all fixed side by side, with their planes vertical and parallel, being supported on a carrier, which can be moved forward in the direction of the waves by means of a rack and pinion underneath. A number of vertical plungers rest in a transverse line with their lower ends resting on the tops of these templets, and are moved up and down as the curves progress forward. The motions of the plungers are then compounded by means of a fine wire passing over pulleys at the top of each one, and under fixed pulleys between adjacent ones. This wire is connected to an indicator, which moves up and down alongside a vertical scale, thus marking the height of the compound wave at any instant.

The wire passing over the plungers is an endless wire, going round a pulley on the indicator and round a larger pulley at the other end of the line of plungers. This larger pulley is attached to a plate which is movable backwards and forwards by means of a fine screw. This gives a means of adjusting the height of the indicator, and also of allowing for the effect of the annual and semi-annual tides. The rise or fall due to these long period tides is treated as constant for fourteen days, and the screw adjusted so as to alter the height of the indicator by the proper amount at the end of each such interval. In front of the frame of the machine, between it and the indicator, is a vertical slide, which is moved forward at the same rate as the carrier, and NO. 1,62 , vOL. 68$]$ carries a sheet of paper on which the tidal curve may be traced if required (Fig. 2).

Each templet is fixed in the carrier in proper relative position according to its phase at the start, as determined by previous harmonic analysis. When the handle of the machine is turned, the carrier, vertical slide and clock are set in motion, and the indicator shows the height of the

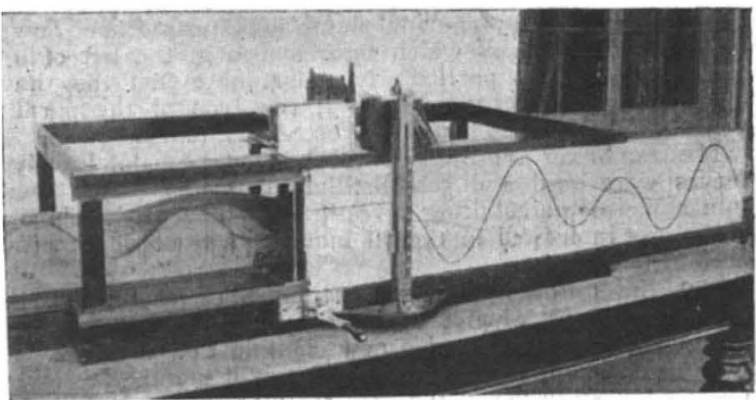

FlG. 2.

tide at the time shown by the clock, and the curve may at the same time be traced on the vertical slide.

There are three carriers and three or four templets to each component. When one of the carriers has been worked forward far enough, it can be disconnected from the others and connected up again at the other end. The curves are again placed in their respective grooves, and, by means of a suitable attachment, butted close up to the preceding ones. In this way the process is made continuous.

The setting of the curves can easily be checked at every month, to see that there has been no slipping.

The machine involves no expensive construction, and enables a year's tides to be predicted expeditiously, and, as experience has proved, with quite sufficient accuracy.

The University, Adelaide.

R. W. Chapman.

[In a subsequent letter, Mr. Chapman informs us that he made the following errors in the list of values of the tidal components at Port Darwin, printed in last week's Nature (p. 295). "The amplitude of N should be $\mathrm{x} \cdot 04$, of $\nu 0.48$, and of T 1.53 . The phase of $\nu$ should be $141^{\circ}$, and that of $T 70^{\circ}$."-Ed. NATURE.]

\section{Sympathetic Song in Birds.}

Is your issue of April 30 (vol. 1xvii. p. 6o9) Mr. Gegrge Henschel describes an interesting vocal duet between a bullfinch and a canary, and invites contributions to the subject.

In 1893 I obtained a nestling Australian magpie (Gymnorhina tibicen, Latham), and taught it on the flute to pipe the following :-

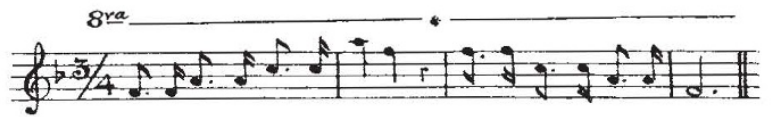

Some years later I acquired another bird of the same species; this learned the tune from the original magpie. I do not know how the birds agreed upon the duet (or fugue) rendering, but it was performed in the following way:When the first bird commenced its song, the second one immediately came to attention, and with half-open beak awaited the point marked *, whence it finished the strain alone. The birds were kept in a large outdoor aviary in company with many others, and no matter where or how engaged, the second bird would, on hearing its mate, assume an attentive attitude, and await the conclusion of the first portion of the theme.

The second bird died, and the original one, which I still have, now pipes the whole strain alone, as was its original custom.

I may also mention that this bird has the faculty of absolute pitch, and pipes the theme in $F$ as originally taught.

Australian Museum, Sydney, June 18.
EDGaR R. WAITE. 